\title{
Reliability and Route Diversity in Wireless Networks
}

\author{
Ehsan Khandani, Eytan Modiano, Jinane Abounadi, Lizhong Zheng ${ }^{1}$ \\ Laboratory for Information and Decision Systems \\ Massachusetts Institute of Technology \\ e-mail: [khandani, modiano,jinane, zheng] @mit.edu
}

\begin{abstract}
-
We study the problem of communication reliability and diversity in multi-hop wireless networks. Our aim is to develop a new network model that better takes into account the fading nature of the wireless physical layer. To that end, we use the outage probability model for a fading channel to develop a probabilistic model for a wireless link. This model establishes a relationship between the link reliability, the distance between communicating nodes and the transmission power. Applying this probabilistic model to a multi-hop network setting, we define and analyze the end-to-end route reliability and develop algorithms for finding the optimal route between a pair of nodes. The relationship between the reliability of the optimal route and the consumed power is studied. The idea of route diversity is introduced as a way to improve the end-to-end route reliability by taking advantage of the wireless broadcast property, the independence of fade state between different pairs of nodes, and the space diversity created by multiple relay nodes along the route. We give analytical results for the improvements due to route diversity in some simple network topologies. Our results suggest that route diversity can fundamentally change the trade off between reliability and power in a multi-hop network.
\end{abstract}

\section{INTRODUCTION}

The area of ad-hoc and sensor networks has received a lot of attention in the research community over the past several years. In this paper, we look at the problem of routing and reliability in these networks.

Motivated by models for the propagation of electromagnetic signals in space, the amount of energy required to establish a link between two nodes is usually assumed to be proportional to the distance between the communicating nodes raised to a constant power. This fixed exponent, referred to as the path-loss exponent, is usually assumed to be between 2 to 4 . In this model, it is assumed that the information is received by the intended destination with certainty if the source transmits the information at a minimum power level dictated by its distance to the intended destination. We refer to this model as the deterministic link model in this paper since the set of nodes that receive the transmitted information is known with certainty based on the transmission power level chosen by the transmitter.

The deterministic model for a wireless link, however, may not be very realistic for describing one of the most important effects in wireless communication, the multi-path fading. The

\footnotetext{
${ }^{1}$ This work was supported by NSF ITR grant CCR-0325401, by DARPA/AFOSR through the University of Illinois grant no. F49620-02-1-0325 and by NASA Space Communication Project grant number NAG3-2835.
}

received signal in a wireless link is the sum of signals reflected by different scatterers in the propagation environment. A link is said to be in a deep fade state when the reflected signals add destructively at the receiver. Naturally, a higher transmission power is required to establish a link between two nodes when the channel between them is in deep fade. Since the fade state of a link changes over time, the amount of energy required to transfer a unit of information between any two nodes changes over time as well. The simple deterministic model for a wireless link does not take into account this time varying nature of the wireless propagation medium.

Depending on how fast channel changes occur, the time varying nature of the wireless channel can be addressed in two different ways. If the channel changes relatively fast, coding can be done to average the effect of fading. This type of averaging effect is the motivation behind the ergodic capacity model for fading channels (see [9]). To achieve this type of average behavior, however, long delays might be imposed on the transmission. In situations where the ergodic capacity is achievable, the deterministic model for the wireless link may still be applicable with some minor changes. We will not get into the details here as our goal in this paper is to propose an alternative model for a wireless link which is more suitable for scenarios in which this type of average behavior is not appropriate. The case of delay sensitive data and slowly changing channel is one example.

An alternative model for the wireless link is based on the outage probability formulation, see [9], [5], and [6]. In this model, the instantaneous capacity of a wireless link is treated as a random variable. A link is said to be in outage when the instantaneous capacity supported by the link is less than the transmission rate. The reliability of a link, i.e. the probability of correct reception at the receiver, is modeled as a function of the transmission rate, the transmitted power, the distance between the communicating nodes, and the channel fade state. In this paper, we assume that the fade state is not known to the transmitter. Under this setting, the transmitter can control the probability of successful reception at its intended receiver by adjusting the transmission rate or power. We refer to this model as the probabilistic link model.

There are several ways to avoid losing data when the channel is in outage, such as coding over a long period of time, employing ARQ protocols, or obtaining transmitter side channel information. However, in this study, we focus on the reliability of a link without using any of these techniques. This approach allows us to isolate the issue of obtaining diversity through routing, and the results developed here can be readily applied in combination with other forms of diversity techniques.

Our analysis starts by looking at the reliability of a pointto-point communication link.

In section II, we develop a model that relates the channel fade state and the distance between the communicating nodes to the probability of successful reception. This would give us 
the mathematical formulation for the probabilistic link model.

In section III, we extend the probabilistic link model to a network setting. In a network setting, we first define and analyze the reliability for a fixed route and then develop algorithms for finding the optimal route between a sourcedestination pair of nodes. The trade-off between route reliability and consumed power is studied. To our knowledge, this is the first attempt to introduce the concept of route reliability and the end-to-end reliability versus power trade-off in a network setting. More precisely, this is the first time that network layer routing algorithms and route properties, such as reliability and power, are studied based on the outage probability model at the physical layer. This model has the potential to open the door for a wide-range of research on wireless network reliability.

In section IV, we introduce the idea of route diversity as a way to improve route reliability by taking advantage of the wireless broadcast property and the independence of fade state between different pairs of nodes. We give analytical results on improvements due to route diversity in some simple network topologies and show how route diversity can fundamentally change the trade-off between the route reliability and the consumed power.

The idea of route diversity is motivated by the work done in [1], [2], [3], and [4]. Most pervious results have been focused on two-hop networks, and the analysis has been based on the information theory results for relay channels. References [1], [2], and [3] look at the effect of cooperation among nodes in increasing the capacity or reducing the outage probability in a fading network. In [1] and [3], the authors described several protocols for taking advantage of this type of diversity in an ad-hoc network. They look at the trade-off between the capacity and the outage improvement in a two-hop network. This analysis ignores the deterministic part of link attenuation due to the distance between nodes and assumes all link fading factors are independent and identically distributed Rayleigh random variables. While [4] looks at the asymptotic benefit of relay nodes in improving the capacity in an ad-hoc network. Their analysis only takes into account the deterministic part of link attenuation due to the distance between nodes. Their results mainly deal with how the capacity scales as a function of the number of nodes in the network.

\section{Probabilistic Link Model}

In this section, we develop the analytical framework for the probabilistic link model. This framework determines the relationship between the probability of successful reception, the distance between the communicating nodes, and the transmission power in a point-to-point single-user flat Rayleigh fading link. We model the received signal as:

$$
\mathrm{y}=\mathrm{a} \mathrm{x}+\eta,
$$

where $\mathrm{x}$ is the transmitted signal, $\eta$ is the additive received noise, $a$ is the signal attenuation due to propagation in the wireless point-to-point link, and y is the received signal. We assume the received noise, $\eta$, is zero mean additive white Gaussian noise with average power of $\sigma_{\eta}^{2}$. In general, attenuation, a, depends on the distance between the communicating nodes and the fade state of the channel. We use $d$ to represent the distance between the communicating nodes and $f$ to represent the fading state of the channel. To emphasize this dependence, we express a explicitly as a function of these two parameters:

$$
\mathrm{y}=\mathrm{a}(\mathrm{f}, \mathrm{d}) \mathrm{x}+\eta \text {. }
$$

In a system with mobile nodes and a constantly changing propagation environment, both $f$ and $d$ change over time. However, we assume a system where $f$ and $d$ remain constant for a long period of time compared to a typical transmission block length. Furthermore, we assume that the transmission blocks are long enough that coding can be done to average over the Gaussian noise. Given these assumptions, the link between two nodes is a simple AWGN channel and the capacity, i.e. the amount of information that can be reliably transmitted through this channel (see[11]), is given by:

$$
\mathrm{C}\left(\mathrm{f}, \mathrm{d},|\mathrm{x}|^{2}, \sigma_{\eta}^{2}\right)=\log \left(1+\frac{|\mathrm{a}(\mathrm{f}, \mathrm{d})|^{2}|\mathrm{x}|^{2}}{\sigma_{\eta}^{2}}\right)
$$

To simplify this notation, we decompose $a(f, d)$ into two independent components corresponding to the small scale fading and the large scale path loss (see [10]). More specifically, we assume:

$$
|\mathrm{a}(\mathrm{f}, \mathrm{d})|^{2}=\frac{|\mathrm{f}|^{2}}{\mathrm{~d}^{\mathrm{k}}}
$$

where $\mathrm{k}$ is the propagation power loss exponent, usually assumed to be between 2 to 4 . Simplifying the capacity formula using this form for a, and simplifying the notation by using $\frac{|x|^{2}}{\sigma_{\eta}^{2}}=$ snr, we get:

$$
C(f, d, s n r)=\log \left(1+\frac{|f|^{2}}{d^{k}} \text { snr }\right)
$$

\section{II.A Outage Formulation}

Eq. (5) gives the instantaneous capacity of the point-topoint link defined by (2). An outage event is said to have occurred (see [5]) when the transmission rate, $\mathrm{R}$ bits/channeluse, is above the instantaneous capacity of the link, i.e.

$$
\{\text { Outage }\} \stackrel{\text { def }}{=}\{C(f, d, \text { snr })<R\} \text {. }
$$

One parameter of interest in communication systems is the probability of error at the receiver. An error occurs if the channel is in outage or if the channel is not in outage but there is a decoding error. In our analysis, we assume that the probability of decoding error is almost zero when channel is not in outage. Under this assumption, outage is the dominating error event. Hence:

$$
\mathrm{P}_{\text {Error }} \approx \mathrm{P}(\text { Outage })
$$

We focus our attention on calculating the outage probability. Based on the definition given in (6), the outage probability is given by:

$$
\begin{aligned}
P_{\text {Outage }} & =\mathrm{P}\{\mathrm{C}(\mathrm{f}, \mathrm{d}, \text { snr })<\mathrm{R}\} \\
& =\mathrm{P}\left\{\log \left(1+\frac{|\mathrm{f}|^{2}}{\mathrm{~d}^{\mathrm{k}}} \mathrm{snr}\right)<\mathrm{R}\right\} \\
& =\mathrm{P}\left\{\frac{|\mathrm{f}|^{2}}{\mathrm{~d}^{\mathrm{k}}}<\frac{2^{\mathrm{R}}-1}{\mathrm{snr}}\right\} .
\end{aligned}
$$

Similar to the approach taken in [1] and other works in this area, we normalize the transmission rate by absorbing its effect into the snr term. So we define:

$$
\mathrm{snr}_{\text {norm } 1}=\frac{\mathrm{snr}}{2^{\mathrm{R}}-1} \text {. }
$$


Equation (7) simplifies to:

$$
P_{\text {Outage }}=P\left\{\frac{|f|^{2}}{d^{k}}<\frac{1}{\text { snr norm1 }_{\text {no }}}\right\}
$$

For the case that fading, $\mathrm{f}$, is random and distance, $\mathrm{d}$, is known to the transmitter, the outage probability simplifies to:

$$
\begin{aligned}
P_{\text {Outage }} & =P\left(\frac{|f|^{2}}{d^{k}}<\frac{1}{s n r_{\text {norm } 1}}\right) \\
& =F_{|f|^{2}}\left(\frac{d^{k}}{s n r_{\text {norm } 1}}\right) .
\end{aligned}
$$

where $F_{|f|^{2}}$ is the CDF of $|f|^{2}$. In our analysis, we model fading as a Rayleigh random variable. For Rayleigh fading with $\mathrm{E}\left[|\mathrm{f}|^{2}\right]=\mu$, the $\mathrm{CDF}$ is given by:

$$
\mathrm{F}_{|\mathrm{f}|^{2}}(\mathrm{x})=1-\exp \left(-\frac{\mathrm{x}}{\mu}\right)
$$

Hence:

$$
\mathrm{P}_{\text {Outage }}=1-\exp \left(-\frac{\mathrm{d}^{\mathrm{k}}}{\mu \mathrm{snr}_{\mathrm{norm} 1}}\right) .
$$

To simplify the notation, we can absorb the effect of $\mu$ into the value of snr $_{\text {norm1 }}$ by defining snr $_{\text {norm2 } 2}$ as:

$$
\mathrm{snr}_{\text {norm2 }}=\mu \mathrm{snr}_{\text {norm } 1} .
$$

For notational convenience, we drop the subscript in the subsequent analysis. The probability of successful reception, or equivalently the reliability, for a Rayleigh fading link with fixed distance is given by the following simple expression:

$$
P_{\text {Succ }}(d, \text { snr })=\exp \left(-\frac{d^{k}}{s n r}\right) \text {. }
$$

Since snr is related to the transmission power, through several levels of normalization to take into account the noise power and the fading parameter, $\mu$, we use this quantity as a proxy for the consumed power in this paper. Furthermore, we assume that the noise power and the fading parameter are constant across the network, hence, in the network scenario that will be discussed shortly, the total snr can be used as a proxy for the total consumed power.

\section{II.B Link Outage-Power Trade-OfF}

Using (11) to find the expression for link outage probability, we have:

$$
\begin{aligned}
\operatorname{P}_{\text {Outage }}(\mathrm{d}, \mathrm{snr}) & =1-\exp \left(-\frac{\mathrm{d}^{\mathrm{k}}}{\mathrm{snr}}\right) \\
& \approx \frac{\mathrm{d}^{\mathrm{k}}}{\mathrm{snr}} .
\end{aligned}
$$

where the approximation is valid for high enough values of snr such that $\frac{d^{k}}{s n r}$ is small. For a point to point link with a single transmitting and receiving antenna, it is known that the outage probability decays as $\mathrm{snr}^{-1}$ in the high-snr regime. See [8] for advanced coverage of this topic and the effect of multiple antennas on this relationship. In the next section, we look at a similar relationship for a multi-hop route.

\section{Reliability at the Network Layer}

A multi-hop route is a sequence of nodes through which the information is relayed from a source node, s, to a destination node, d, i.e.

$$
\text { Route }=\left(r_{0}, r_{1}, \cdots, r_{h-1}, r_{h}\right)
$$

where, $r_{0}=s, r_{h}=d$, and $h$ is the number of hops. We assume the network operates based on a time division protocol under which successive transmissions along a route happen in consecutive transmission slots. Route $\left(\mathbf{s}, \mathrm{r}_{1}, \cdots, \mathrm{r}_{\mathrm{h}-1}, \mathrm{~d}\right)$ is identical to a sequence of $\mathrm{h}$ point-to-point links, where for the $\mathrm{i}^{\mathrm{th}}$ link, relay $i-1$ is the transmitter and relay $i$ is the receiver, snr $_{r_{i-1}} r_{i}$ is the transmitted signal-to-noise power, and $d_{r_{i-1}} r_{i}$ is the distance between the nodes. We define the event of successful end-to-end transmission as the event that all $\mathrm{h}$ transmissions are successful and the End-to-End Reliability is defined as the probability of this event. We assume that the fading factors for different links are independent and identically distributed Rayleigh random variables. Based on this assumption and using results from (11), the end-to-end reliability can be written as:

$$
\begin{aligned}
\text { Reliability }^{\left(r_{0}, r_{1}, \cdots, r_{h-1}, r_{h}\right)} & =\prod_{i=1}^{h} \exp \left(-\frac{d_{r_{i-1} r_{i}}^{k}}{s n r_{r_{i}-1} r_{i}}\right) \\
& =\exp \left(-\sum_{i=1}^{h} \frac{d_{r_{i-1} r_{i}}^{k}}{s n r_{r_{i}-1} r_{i}}\right) .
\end{aligned}
$$

The corresponding total amount of power spent for successful end-to-end transmission is:

$$
\mathrm{SNR}_{\text {Total }}^{\left(r_{0}, r_{1}, \cdots, r_{h-1}, r_{h}\right)}=\sum_{i=1}^{h} \operatorname{snr}_{r_{i-1} r_{i}} .
$$

In the subsequent analysis, we use the route reliability defined by (12) and the route outage probability, $\rho$, given below interchangeably.

$$
\rho^{\left(r_{0}, r_{1}, \cdots, r_{h-1}, r_{h}\right)}=1-\text { Reliability }^{\left(r_{0}, r_{1}, \cdots, r_{h-1}, r_{h}\right)} .
$$

There are three different questions in connection with the end-to-end reliability and power that we consider:

1. What is the end-to-end reliability if the maximum transmitted power per link is fixed?

2. What is the minimum total power required to achieve a guaranteed level of end-to-end reliability?

3. What is the maximum end-to-end reliability for a fixed total power?

The first problem is motivated by the fact that in some cases the transmitted power by each node might be limited due to hardware constraints or to limit the interference level to other nodes. The second problem is a power allocation problem, where the objective is to minimize the total consumed power subject to a guaranteed level of end-to-end reliability. The last problem is also a power allocation problem, where the objective is to maximize the end-to-end reliability of a route subject to a total power constraint. The last two problems might be of interest in cooperative networks where each node is equally likely to be source, destination, or relay of the traffic. In these scenarios, it is reasonable to minimize the total consumed power subject to a reliability constraint, problem 2 , or maximize the end-to-end reliability subject to a total consumed power, i.e. problem 3 . 
1. Maximum End-to-End Reliability for a Fixed Maximum Transmission Power Per Link

Assuming the transmitted signal-to-noise ratio at each link is limited to $\mathrm{SNR}_{\text {Link-Max }}$, the corresponding route reliability can be readily calculated using (12). For a fixed route, $\left(r_{0}, r_{1}, \cdots, r_{h-1}, r_{h}\right)$, the end-to-end reliability is given by:

$$
\text { Reliability }^{\left(r_{0}, r_{1}, \cdots, r_{h-1}, r_{h}\right)}=\exp \left(-\frac{\sum_{i=1}^{h} d_{r_{i}-1}^{k} r_{i}}{S N R_{L i n k}-M a x}\right) .
$$

According to this expression, the end-to-end reliability is a monotonically decreasing function of $\sum_{i=1}^{h} d_{r_{i}-1}^{k} r_{i}$. This quantity can be treated as the cost metric for route selection. The most reliable route between two nodes is the route that minimizes this cost metric. We refer to route selection algorithm based on this cost metric as the Minimum $\mathbf{O}$ utage $\mathbf{R}$ oute, MOR, algorithm.

Lemma 1 The most reliable route between nodes $\mathbf{s}$ and $\mathrm{d}$ in a fixed multi-hop wireless network where the fading parameters of different links are independent Rayleigh random variables and the maximum transmitted snr at each node is limited to $\mathrm{SNR}_{\mathrm{Max}-\mathrm{Link}}$ is the route

$$
\left(s, r_{1}, \cdots, r_{h-1}, d\right)=\left(r_{0}, r_{1}, \cdots, r_{h-1}, r_{h}\right)
$$

that minimizes

$$
\sum_{i=1}^{h} d_{r_{i-1} r_{i}}^{k}
$$

and the reliability of this route is given by (15).

\section{Minimum End-to-End Power for a Guaranteed End-to-End Reliability}

The problem of minimizing the end-to-end power for a fixed route, $\left(r_{0}, r_{1}, \cdots, r_{h-1}, r_{h}\right)$, and fixed end-to-end reliability, Reliabilitymin, is formulated as the following constrained optimization problem:

$$
\begin{array}{ll}
\min & \sum_{i=1}^{h} s n r_{r_{i}-1} r_{i} \\
\text { s.t. } & \exp \left(-\sum_{i=1}^{h} \frac{d_{r_{i-1} r_{i}}^{k}}{s n r_{r_{i}-1} r_{i}}\right) \geq \text { Reliability }_{M i n} .
\end{array}
$$

Since exponential is a monotonically increasing function, the constraint must be satisfied with equality at the optimal solution. So, the optimization problem is equivalent to:

$$
\begin{array}{ll}
\min & \sum_{i=1}^{h} s n r_{r_{i-1} r_{i}} \\
\text { s.t. } & \sum_{i=1}^{h} \frac{d_{r_{i-1} r_{i}}^{k}}{s n r_{r_{i}-1} r_{i}}=-\ln \left(\text { Reliability }_{M i n}\right) .
\end{array}
$$

The Lagrangian for this problem is given by:

$$
\begin{aligned}
& \mathrm{L}\left(\mathrm{snr}_{\mathrm{r}_{0} \mathrm{r}_{1}}, \cdots, \mathrm{snr}_{\mathrm{r}_{\mathrm{h}-1} \mathrm{r}_{\mathrm{h}}}, \lambda\right) \\
& =\sum_{\mathrm{i}=1}^{\mathrm{h}} \operatorname{snr}_{\mathrm{r}_{\mathrm{i}-1} \mathrm{r}_{\mathrm{i}}}+\lambda\left(\sum_{\mathrm{i}=1}^{\mathrm{h}} \frac{\mathrm{d}_{\mathrm{r}_{\mathrm{i}-1} \mathrm{r}_{\mathrm{i}}}^{\mathrm{k}}}{\text { snr }_{\mathrm{r}_{\mathrm{i}-1} \mathrm{r}_{\mathrm{i}}}}+\ln \left(\text { Reliability }_{\text {Min }}\right)\right) \text {. }
\end{aligned}
$$

The partial derivatives with respect to the transmitted snr at each intermediate relay is:

$$
\frac{\partial \mathrm{L}}{\partial s n r_{r_{i}-1} r_{i}}=1-\lambda \frac{d_{r_{i-1} r_{i}}^{k}}{s n r_{r_{i}-1}^{2} r_{i}} .
$$

Setting these first order conditions to 0 and solving for the optimal transmitted snr, we get:

$$
\widehat{\operatorname{snr}}_{r_{0} r_{1}}=\sqrt{\lambda d_{r_{i}-1}^{k} r_{i}}
$$

Substituting these into the constraint and solving for the optimal $\lambda$, we get:

$$
\sqrt{\widehat{\lambda}}=\frac{\sum_{i=1}^{h} \sqrt{d_{r_{i}-1 r_{i}}^{k}}}{-\ln (\text { Reliability } \operatorname{Min})} .
$$

Substituting this back into (18), the optimal transmitted signal-to-noise ratio for each node is given by:

$$
\widehat{\operatorname{snr}} r_{r_{i-1}} r_{i}=\frac{\sum_{i=1}^{h} \sqrt{d_{r_{i}-1}^{k} r_{i}}}{-\ln (\text { ReliabilityMin })} \sqrt{d_{r_{i-1} r_{i}}^{k}} .
$$

The resulting optimal end-to-end power is given by:

$$
\begin{aligned}
& \widehat{\operatorname{SNR}}_{\text {Total }}=\sum_{\mathrm{i}=1}^{\mathrm{h}} \widehat{\operatorname{snr}}_{\mathrm{r}_{\mathrm{i}-1} \mathrm{r}_{\mathrm{i}}} \\
& =\sum_{i=1}^{h}\left(\frac{\sum_{j=1}^{h} \sqrt{d_{r_{j}-1}^{k} r_{j}}}{-\ln \left(\text { Reliability } y_{\text {Min }}\right)}\right) \sqrt{d_{r_{i-1} r_{i}}^{k}} \\
& =\frac{\left(\sum_{i=1}^{h} \sqrt{d_{r_{i}-1 r_{i}}^{k}}\right)^{2}}{-\ln \left(\text { Reliability }{ }_{M i n}\right)} \text {. }
\end{aligned}
$$

For easier future reference, we state this result in lemma 2.

Lemma 2 For a fixed route $\left(\mathrm{r}_{0}, \mathrm{r}_{1}, \cdots, \mathrm{r}_{\mathrm{h}-1}, \mathrm{r}_{\mathrm{h}}\right)$, the minimum required total power to guarantee the end-toend reliability of Reliabilitymin is

$$
\widehat{S N R}_{\text {Total }}=\frac{\left(\sum_{i=1}^{h} \sqrt{d_{r_{i}-1 r_{i}}^{k}}\right)^{2}}{-\ln (\text { ReliabilityMin })},
$$

which is achieved when the transmission powers are allocated such that

$$
\widehat{\operatorname{snr}}_{r_{i-1} r_{i}}=\frac{\sum_{i=1}^{h} \sqrt{d_{r_{i}-1}^{k} r_{i}}}{-\ln (\text { ReliabilityMin })} \sqrt{d_{r_{i}-1}^{k} r_{i}} .
$$

From lemma 2, we know that for any route, $\left(r_{0}, r_{1}, \cdots, r_{h-1}, r_{h}\right)$, and under optimal power allocation scheme, the total power required to achieve a desired level of end-to-end reliability is a monotonically increasing function of $\sum_{i=1}^{h} \sqrt{d_{r_{i-1} r_{i}}^{k}}$. Hence, the minimum power route is the route among all possible routes between two nodes that minimizes this sum. We refer to this route selection scheme as the Minimum $\mathbf{E}$ nergy Route, MER, algorithm.

Theorem 1 In a multi-hop wireless network where the fading parameters for different links are independent Rayleigh random variables, the minimum power route 
between nodes $\mathrm{s}$ and $\mathrm{d}$ subject to the guaranteed end-toend reliability of Reliability Min $_{\text {is }}$ the route

$$
\left(\mathrm{s}, \mathrm{r}_{1}, \cdots, \mathrm{r}_{\mathrm{h}-1}, \mathrm{~d}\right)=\left(\mathrm{r}_{0}, \mathrm{r}_{1}, \cdots, \mathrm{r}_{\mathrm{h}-1}, \mathrm{r}_{\mathrm{h}}\right)
$$

that minimizes

$$
\sum_{\mathrm{i}=1}^{\mathrm{h}} \sqrt{\mathrm{d}_{\mathrm{r}_{\mathrm{i}-1} \mathrm{r}_{\mathrm{i}}}^{\mathrm{k}}}
$$

and the corresponding end-to-end power is given by (21).

\section{Maximum End-to-End Reliability for a Fixed End-to-End Power}

The problem of achieving maximum end-to-end reliability for a fixed route, $\left(r_{0}, r_{1}, \cdots, r_{h-1}, r_{h}\right)$, and fixed endto-end power, SNR $\mathrm{S}_{\text {Total-Max }}$, can also be formulated as a constrained optimization problem:

$$
\begin{aligned}
& \max \quad \exp \left(-\sum_{i=1}^{h} \frac{d_{r_{i-1} r_{i}}^{k}}{\operatorname{snr}_{r_{i-1} r_{i}}}\right) \\
& \text { s.t } \quad \sum_{i=1}^{h} \text { snr }_{r_{i-1} r_{i}} \leq \text { SNR Rotal-Max }_{\text {Tol }} .
\end{aligned}
$$

This problem can be solved using a technique very similar to the approach used to solve (16). Skipping the details of the optimization solution, we simply present the solution to $(22)$ in lemma 3 .

Lemma 3 For a fixed route $\left(\mathrm{r}_{0}, \mathrm{r}_{1}, \cdots, \mathrm{r}_{\mathrm{h}-1}, \mathrm{r}_{\mathrm{h}}\right)$ and for a fixed end-to-end power of $\mathrm{SNR}_{\mathrm{Total}-\mathrm{Max}}$, the maximum end-to-end reliability is

$$
\text { Reliabilityoptimal }=\exp \left(-\frac{\left(\sum_{\mathrm{i}=1}^{\mathrm{h}} \sqrt{\mathrm{d}_{\mathrm{r}_{\mathrm{i}-1} \mathrm{r}_{\mathrm{i}}}^{\mathrm{k}}}\right)^{2}}{\mathrm{SNR} \mathrm{R}_{\text {Total }- \text { Max }}}\right),
$$

and the optimal power allocation that achieves this reliability is

$$
\widehat{\operatorname{snr}}_{r_{i-1} r_{i}}=S N R_{\text {Total }-M a x} \frac{\sqrt{d_{r_{i}-1}^{k} r_{i}}}{\sum_{i=1}^{h} \sqrt{d_{r_{i}-1}^{k} r_{i}}} .
$$

From lemma 3, we know for any route, $\left(r_{0}, r_{1}, \cdots, r_{h-1}, r_{h}\right)$, and under the optimal power allocation scheme the end-to-end reliability is a monotonically decreasing function of $\sum_{i=1}^{h} \sqrt{d_{r_{i}-1}^{k} r_{i}}$. Hence, the maximum reliability route is the route that minimizes this sum. We state this result in the following theorem

Theorem 2 The most reliable route between nodes $\mathrm{s}$ and $\mathrm{d}$ in a fixed multi-hop wireless network where the fading parameters of different links are independent Rayleigh random variables and the maximum end-to-end power is limited to $\mathrm{SNR}_{\text {Total-Max }}$ is the route

$$
\left(s, r_{1}, \cdots, r_{h-1}, d\right)=\left(r_{0}, r_{1}, \cdots, r_{h-1}, r_{h}\right)
$$

that minimizes

$$
\sum_{i=1}^{h} \sqrt{d_{r_{i}-1}^{k} r_{i}},
$$

and the corresponding end-to-end reliability is given by (23).

\section{III.A Optimal Reliability-Power Curve}

Two optimization problems that we looked at in the last section, formulated in (16) and (22), are in fact dual problems. Hence, it is not surprising that the cost metric in both cases turned out to be $\sum_{i=1}^{h} \sqrt{d_{r_{i-1} r_{i}}^{k}}$. To clarify this point, we present a graphical illustration of the relationship between the end-to-end reliability and power under the optimal power allocation scheme.

For any fixed route, different power allocation schemes result in different end-to-end reliability and consumed power. If we were to characterize each power allocation scheme only by the total consumed power and the resulting end-to-end reliability, each allocation scheme could be represented by a point in the two dimensional plot of the end-to-end reliability vs. the total power. Certain allocation schemes are optimal, i.e. either minimize the total power consumed to achieve a guaranteed end-to-end reliability or maximize the end-to-end reliability for a fixed consumed power.

In problem 2, we found the optimal power allocation to minimizes the total power subject to a guaranteed end-toend reliability. Graphically, this optimization corresponds to moving along the horizontal line in figure 1 and finding the allocation scheme that minimizes the total consumed power subject to an end-to-end reliability of Reliability $\min _{\text {. }}$. We found that the reliability and power corresponding to the optimal allocation are related by the following relationship:

$$
\widehat{S N R}_{\text {Total }}=\frac{\left(\sum_{i=1}^{h} \sqrt{d_{r_{i-1} r_{i}}^{k}}\right)^{2}}{-\ln (\text { ReliabilityMin })} .
$$

In problem 3, we found the optimal power allocation to maximize the end-to-end reliability for a given end-to-end power. This corresponds to moving along the vertical line in figure 1 and finding the allocation scheme that maximizes the reliability for SNR $\mathrm{R}_{\text {Total-Max }}$ We found that the resulting end-to-end reliability for this optimal allocation is:

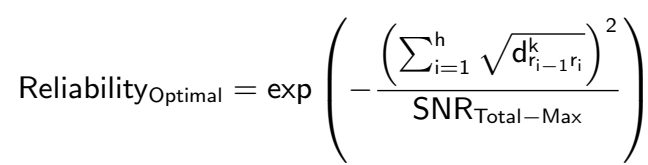

Clearly, the curve specified by (24) and (25) are identical. The set of optimal power allocations can be represented by a single curve in the two dimensional plot of the end-to-end reliability vs. total power as shown in figure 1. We refer to this curve as the Optimal Reliability-Power Trade-off curve.

\section{III.B Route Outage-Power Trade-OFF}

Similar to the case of a point-to-point link, it is insightful to look at the trade-off between route outage and the consumed power. This type of analysis gives insight to the required power to achieve a desired end-to-end outage level or how fast the end-to-end outage dacays with power.

For the case that the maximum transmitted power at each link is limited to $\mathrm{SNR}_{\text {Max-Link, }}$ (15) gives the end-to-end reliability. We get more insight by looking at the route outage probability defined in (14). Writing (15) in terms of the outage probability, $\rho$, we have:

$$
\begin{aligned}
1-\rho & =\exp \left(-\frac{\sum_{i=1}^{h} d_{r_{i}-1}^{k} r_{i}}{S N R_{\text {Max }-L i n k}}\right), \\
\ln (1-\rho) & =-\frac{\sum_{i=1}^{h} d_{r_{i-1} r_{i}}^{k}}{S N R_{\text {Max }- \text { Link }}} .
\end{aligned}
$$




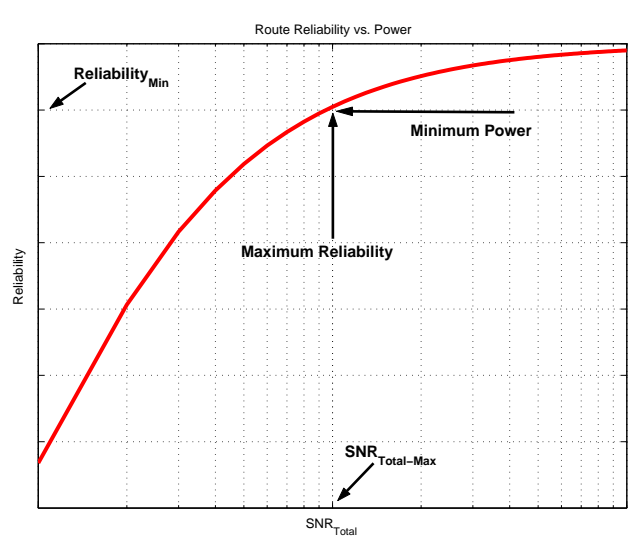

Figure 1: Route Reliability vs. Power

For small values of $\rho$, we can use the approximation of $\ln (1-\rho) \approx-\rho$ to simplify this relation to:

$$
\rho \approx \frac{\sum_{\mathrm{i}=1}^{\mathrm{h}} \mathrm{d}_{\mathrm{r}_{\mathrm{i}-1} \mathrm{r}_{\mathrm{i}}}^{\mathrm{k}}}{\text { SNR }_{\operatorname{Max}-\text { Link }}} .
$$

The relationship between reliability and power with optimal power allocation, i.e. the optimal reliability-power curve discussed in the last section, is given by (25). Writing (25) in terms of the route outage probability and following a similar approach, we find:

$$
\rho \approx \frac{\left(\sum_{\mathrm{i}=1}^{\mathrm{h}} \sqrt{\mathrm{d}_{\mathrm{r}_{\mathrm{i}-1} \mathrm{r}_{\mathrm{i}}}^{\mathrm{k}}}\right)^{2}}{\mathrm{SNR}_{\text {Total }}} .
$$

From (26) and (27), we observe that route outage decays as $\mathrm{SNR}_{\text {Max }- \text { Link }}^{-1}$ and $\mathrm{SNR}_{\text {Total }}^{-1}$, respectively, in the high-snr regimes. It is not surprising that we observe this type of relation as these relationships are very similar to what we observed in the first section for a point-to-point link. In the last section, we see how diversity at the route level can fundamentally change this trade-off.

\section{Route Diversity}

So far in this paper, we have taken an approach in which a multi-hop route is treated as a single end-to-end pipe. Under this scenario, a successful end-to-end reception requires all point-to-point transmissions to be successful. Even in this very limited scheme, it possible to improve the end-to-end route reliability by taking advantage of the wireless broadcast property and the independence between different Rayleigh fading links. This is the motivation behind the Route Diversity idea that we introduce in this section.

To clarify this idea, let's look at a simple example. Assume that in the network shown in figure 2 , the most reliable route is selected as shown. Without diversity, a successful end-to-end relaying require 3 successful point-to-point transmissions. We refer to this strategy as the Non-Diversified Routing Scheme. Due to the broadcast and the fading nature of the wireless propagation environment, the information transmitted by $\mathrm{s}$ may be received correctly by, for example, $r_{1}$ while $r_{0}$ fails to receive that information. By accounting for this possibility, as shown in figure $3, \mathrm{~d}$ can receive the information directly from $s$ in the first transmission slot, from $r_{0}$ in the second transmission slot or from $r_{1}$ in the third transmission slot. We refer

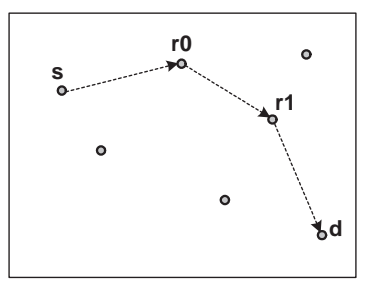

Figure 2: Simple Route

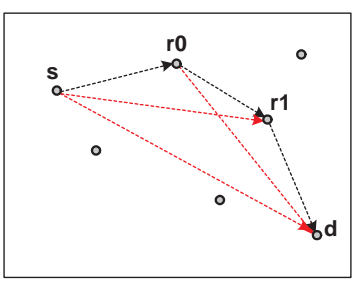

Figure 3: Diversified Route to this routing scheme as the Diversified Routing Scheme. In the subsequent analysis, we assume that the maximum transmitted snr by each node is fixed. This is the quantity we represented by $S_{N R} R_{\text {Max-Link }}$ in the previous section. However, for simpler notation, we drop the subscript and denote this quantity as snr in the analysis that follows. Our aim is to find how the end-to-end outage probability varies with the maximum transmitted power level under the diversified routing scheme and compare the result with the relation given in (26). To further simplify the analysis, we also assume that the path-loss exponent, $\mathrm{k}$, is 2. Many of our results can be extended to have $k$ as a parameter. We study the benefit of route diversity by looking at two examples.

\section{IV.A ExAmple 1: Two Hop-NeTworks}

This example focuses on a 2-hop network constructed by uniformly placing a relay node inside a circle with radius of 1 centered at the mid-point between s and d, figure 4. Based

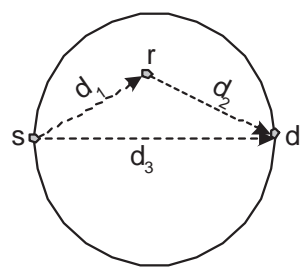

Figure 4: 2-Hop Disk Network

on the results from Lemma 1, the Minimum Outage Route (MOR) in this network is $(s, r, d)$. The non-diversified outage probability for this route is:

$$
\begin{aligned}
\rho_{\text {Non-Diversified }}^{(\mathrm{s}, \mathrm{r}, \mathrm{d})} & =1-\mathrm{P}_{\text {Succ }}\left(\mathrm{d}_{1}, \mathrm{snr}\right) \mathrm{P}_{\text {Succ }}\left(\mathrm{d}_{2}, \mathrm{snr}\right) \\
& \approx \frac{\mathrm{d}_{1}^{2}+\mathrm{d}_{2}^{2}}{\mathrm{snr}}
\end{aligned}
$$

where the approximation is valid in the high-snr regime. In the diversified scheme, successful relaying requires either a successful direct transmission, $\{\mathrm{s} \rightarrow \mathrm{d}\}$, or successful multihoping, $\{s \rightarrow r\}$ followed by $\{r \rightarrow d\}$. The reliability of this route is given by:

$$
\begin{aligned}
\text { Reliability }_{\text {Diversified }}^{(\mathrm{s}, \mathrm{d})}= & \mathrm{P}_{\text {Succ }}\left(\mathrm{d}_{3}, \mathrm{snr}\right)+\left(1-\mathrm{P}_{\text {Succ }}\left(\mathrm{d}_{3}, \mathrm{snr}\right)\right) \\
& \mathrm{P}_{\text {Succ }}\left(\mathrm{d}_{1}, \mathrm{snr}\right) \mathrm{P}_{\text {Succ }}\left(\mathrm{d}_{2}, \mathrm{snr}\right), \\
= & \exp \left(-\frac{\mathrm{d}_{3}^{2}}{\mathrm{snr}}\right)+
\end{aligned}
$$




$$
\begin{aligned}
& \left(1-\exp \left(-\frac{d_{3}^{2}}{s n r}\right)\right) \exp \left(-\frac{d_{1}^{2}+d_{2}^{2}}{s n r}\right) \\
\approx & 1-\frac{d_{3}^{2}\left(d_{1}^{2}+d_{2}^{2}\right)}{s n r^{2}} .
\end{aligned}
$$

where the approximation is valid in the high-snr regime. Hence, the route outage probability is simply:

$$
\rho_{\text {Diversified }}^{(\mathrm{s}, \mathrm{d}, \mathrm{d})} \approx \frac{\mathrm{d}_{3}^{2}\left(\mathrm{~d}_{1}^{2}+\mathrm{d}_{2}^{2}\right)}{\mathrm{snr}^{2}} .
$$

This expression shows that the end-to-end outage probability decays as $\mathbf{s n r}^{-2}$ for the diversified routing scheme. This is a significant improvement over the $\mathrm{snr}^{-1}$ decay observed in (28) in the absence of diversity. It should be noted that both (28) and (30) are valid for any two hop network. Furthermore, this gain is achieved through route diversity and does not require any coding, ARQ, or transmitter side information.

\section{IV.B Example 2: Disconnect Probability}

Consider a network in which nodes are distributed on a line and the distance between neighboring nodes are independent exponential random variables with parameter $\lambda$. Assume that the destination is located a large number of hops away to the right of the source node. Although it may be possible to calculate the exact end-to-end outage probability as a function of the maximum transmitted power level, the location of the relay nodes, and the number of hops, we will take a different approach in analyzing the benefit of route diversity in this network. We define the disconnect event for a node as the event that the node is not connected to any node located to its right. Without diversity, this event is equivalent to the event that the link between the node and its immediate right neighbor is in outage, see figure 5. With diversity, however, this event is equivalent to the event that all the links between the node and all the nodes to its right are in outage, see figure 6. Clearly the second event has a lower probability as it is a subset of the first event. We are interested in analytically calculating these probabilities and observing how these quantities depends on the snr.

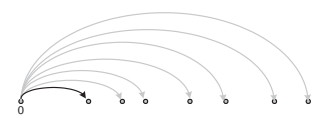

Figure 5: Disconnect, NoDiversity

For a given network realization, i.e. given the distance to the neighboring node is $d_{r}$, the probability of the disconnect event without diversity is given by:

$$
\begin{aligned}
P_{\text {Disconnect }}^{\text {No-Diversity }}\left(\text { snr, } d_{r}\right) & =1-P_{\text {Succ }}\left(d_{r}, \text { snr }\right) \\
& =\exp \left(\frac{-d_{r}^{2}}{s n r}\right)
\end{aligned}
$$

Calculating the probability of the disconnect event with diversity requires a different approach. We start by dividing the line into segments of length $\delta$. For small values of $\delta$, the number of nodes in a line segment of length $\delta$ is approximately a Bernoulli random variable, i.e. there is a node in a segment with probability $\lambda \delta$ or there is no node with probability $1-\lambda \delta$. Furthermore, the number of nodes in non-overlapping line segments are independent random variables, see [7] for details. This approximation is prefect for the derivation that follows as we will take the limit of $\delta \rightarrow 0$ to get the desired result. For small values of $\delta$, let's define the disconnect event for segment located at distance $\mathrm{m} \delta$ away from the transmitter as the event that the information is not received by any node located in the line segment $(\mathrm{m} \delta,(\mathrm{m}+1) \delta]$. This event includes both the case that there is no node in this line segment or there is a node and the transmission fails due to bad fading. The probability of this event can be calculated as:

$$
\mathrm{P}_{\text {Disconnect }}(\mathrm{m} \delta, \mathrm{snr})=1-\mathrm{P}_{\text {Succ }}(\mathrm{m} \delta, \mathrm{snr}) \lambda \delta
$$

where $\mathrm{P}_{\text {succ }}(\mathrm{d}, \mathrm{snr})$ is given in (11). Let $\mathrm{P}_{\text {Disconnect }}(\mathrm{x}, \mathrm{y}, \delta$, snr) be the probability that the information transmitted by a node located at location 0 is not received by any node between $(x, y]$ where this segment is broken down into segments of length $\delta$. This probability can written in terms of $\mathrm{P}_{\text {Disconnect }}(\mathrm{m} \delta, \mathrm{snr})$ calculated above as:

$$
\mathrm{P}_{\text {Disconnect }}(\mathrm{x}, \mathrm{y}, \delta, \mathrm{snr})=\prod_{\mathrm{i}=\frac{\mathrm{x}}{\delta}}^{\frac{\mathrm{y}}{\delta}} \mathrm{P}_{\text {Disconnect }}(\mathrm{i} \delta, \mathrm{snr})
$$

Taking the natural log of both sides, we get:

$$
\begin{aligned}
\ln \left(\mathrm{P}_{\text {Disconnect }}(\mathrm{x}, \mathrm{y}, \delta, \mathrm{snr})\right) & =\sum_{\mathrm{i}=\frac{\mathrm{x}}{\delta}}^{\frac{\mathrm{y}}{\delta}} \ln \left(\mathrm{P}_{\text {Disconnect }}(\mathrm{i} \delta, \text { snr })\right) \\
& =\sum_{\mathrm{i}=\frac{\mathrm{x}}{\delta}}^{\frac{y}{\delta}} \ln \left(1-\mathrm{P}_{\text {Succ }}(\mathrm{m} \delta) \lambda \delta\right) .
\end{aligned}
$$

Taking the limit $\delta \rightarrow 0$ :

$$
\ln \left(P_{\text {Disconnect }}(x, y, \text { snr })\right)=-\int_{x}^{y} \lambda P_{\text {Succ }}(I, \text { snr }) \mathrm{dl}
$$

where we used the approximation of $\ln (1-x) \approx-x$ for small values of $x$ in the last step. For the case when the path-loss exponent $\mathrm{k}=2$, the above integral can be easily calculated based on the complementary error function:

$$
\begin{aligned}
& \ln \left(P_{\text {Disconnect }}(x, y, s n r)\right) \\
& =-\lambda \sqrt{\operatorname{snr}} \int_{\frac{x}{\sqrt{s n r}}}^{\frac{y}{\sqrt{s n r}}} e^{-t^{2}} d t . \\
& =-\frac{\lambda \sqrt{\pi s n r}}{2}\left(\operatorname{erfc}\left(\frac{y}{\sqrt{s n r}}\right)-\operatorname{erfc}\left(\frac{x}{\sqrt{s n r}}\right)\right)
\end{aligned}
$$

Let $\mathrm{P}_{\text {Disconnect }}(\mathrm{snr})$ be the disconnect probability, i.e. the probability that a node is not connected to any node to its right. Assuming an infinitely long line, this probability is obtained by evaluating (33) for $\mathrm{x}=0$ an $\mathrm{y}=\infty$. We have:

$$
\begin{aligned}
\ln \left(P_{\text {Disconnect }}(\mathrm{snr})\right) & =\ln \left(\mathrm{P}_{\text {Disconnect }}(0, \infty, \mathrm{snr})\right) \\
& =-\frac{\sqrt{\pi \lambda^{2} \mathrm{snr}}}{2}
\end{aligned}
$$

Hence:

$$
\mathrm{P}_{\text {Disconnect }}(\mathrm{snr})=\exp \left(-\frac{\sqrt{\pi \lambda^{2} \mathrm{snr}}}{2}\right)
$$

Comparing (31) and (34), it is clear that without diversity, the disconnect probability decays exponentially with $\mathrm{snr}^{-1}$ while 
with diversity, the disconnect probability decays exponentially with $\sqrt{s n r}$. It should be noted that we arrived at this result without making any high-snr assumption. Furthermore, result is valid for any network realization, which adds to its significance.

\section{Summary And Conclusions}

We studied the problem of route reliability in a multi-hop wireless network. Our analysis started by looking at the reliability of a point-to-point communication link. Based on this analysis, we proposed a new probabilistic way of looking at a wireless link. We used this probabilistic model to look at the reliability in a wireless network. In that context, we first defined and analyzed the reliability for a fixed route and then developed algorithms for finding the optimal route between a source-destination pairs of nodes. We looked at three different formulation for the routing problem: finding the most reliable route for a fixed maximum transmitted snr per link, finding the most reliable route for a fixed end-to-end power, and finding the minimum power route for a guaranteed endto-end reliability. We showed that the last two problems are dual of each other. Based on this duality, we found the optimal trade-off curve between the end-to-end reliability and the end-to-end power consumption. It was shown that the trade-off between the end-to-end reliability and the consumed power in a route is very similar to the trade-off between the transmission power and reliability in a link.

The idea of route diversity was introduced as a way to improve the end-to-end reliability by taking advantage of the wireless broadcast property and the independence of the fade parameters between different pairs of nodes. We gave analytical results for improvements due to route diversity in some simple network topologies.

The model proposed in this paper can open the door to a new area of research on communication reliability at the network layer. The trade-off among different route properties, such as the end-to-end reliability, the delay, or the total consumed power should be studied to help draw a better picture of the actual limits of communication in a multi-hop wireless network. In this context, route diversity appears to have the potential to fundamentally change these trade-offs.

\section{REFERENCES}

[1] J.N. Laneman, "Cooperative Diversity in Wireless Networks: Algorithms and Architectures," Ph.D. Thesis, Massachusetts Institute of Technology, Cambridge, MA, August 2002.

[2] J.N. Laneman, G.W. Wornell, "Energy-efficient antenna sharing and relaying for wireless networks," Wireless Communications and Networking Conference, Sept. 2000, Vol.1, pp 7-12

[3] J.N. Laneman, G.W. Wornell, "Distributed space-time-coded protocols for exploiting cooperative diversity in wireless networks," IEEE Transactions on Information Theory, Oct. 2003

[4] M. Gastpar, M. Vetterli, "On the capacity of wireless networks: the relay case," Proc. INFOCOM 2002, Vol. 3, pp 1577-1586

[5] E. Biglieri, J. Proakis, S. Shamai, "Fading channels: information-theoretic and communications aspects," Information Theory, IEEE Transactions on , Volume: 44, Issue: 6 , Oct. 1998

[6] L.H. Ozarow, S. Shamai, A.D. Wyner, "Information theoretic considerations for cellular mobile radio," IEEE Transactions on Vehicular Technology, May 1994

[7] R. Gallagar, "Discrete Stochastoc Processes," Kluwer Academic Publisher, 1996
[8] Lizhong Zheng, D.N.C Tse, "Diversity and multiplexing: a fundamental tradeoff in multiple-antenna channels," IEEE Transactions on Information Theory, May 2003

[9] D. Tse, P. Viswanath, "Fundamental of Wireless Communications", working draft.

[10] T. S. Rappaport, "Wireless Communications: Principles and Practice", Prentice Hall, 2002.

[11] John G. Proakis, "Digital Communiation," Forth Edition, McGrawHill, 2001

[12] Amir E. Khandani, "Cooperative Routing in Wireless Networks", Master's Thesis, MIT, May 2004 